\title{
Comparison of Swallowing Functions Between Brain Tumor and Stroke Patients
}

\author{
Dae Hwan Park, $\mathrm{MD}^{1}$, Min Ho Chun, $\mathrm{MD}^{1}$, Sook Joung Lee, $\mathrm{MD}^{2}$, Yoon Bum Song, $\mathrm{MD}^{1}$
}

\begin{abstract}
${ }^{1}$ Department of Rehabilitation Medicine, Asan Medical Center, University of Ulsan College of Medicine, Seoul; ${ }^{2}$ Department of Rehabilitation Medicine, Regional Cardiocerebral Rehabilitation Center, Dong-A University College of Medicine, Busan, Korea
\end{abstract}

Objective To compare the swallowing functions according to the lesion locations between brain tumor and stroke patients.

Methods Forty brain tumor patients and the same number of age-, lesion-, and functional status-matching stroke patients were enrolled in this study. Before beginning the swallowing therapy, swallowing function was evaluated in all subjects by videofluoroscopic swallowing study. Brain lesions were classified as either supratentorial or infratentorial. We evaluated the following: the American Speech-Language-Hearing Association (ASHA) National Outcome Measurement System (NOMS) swallowing scale, clinical dysphagia scale, functional dysphagia scale (FDS), penetration-aspiration scale (PAS), oral transit time, pharyngeal transit time, the presence of vallecular pouch residue, pyriform sinus residue, laryngopharyngeal incoordination, premature spillage, a decreased swallowing reflex, pneumonia, and the feeding method at discharge.

Results The incidence of dysphagia was similar in brain tumor and stroke patients. There were no differences in the results of the various swallowing scales and other parameters between the two groups. When compared brain tumor patients with supratentorial lesions, brain tumor patients with infratentorial lesions showed higher proportion of dysphagia $(\mathrm{p}=0.01)$, residue $(\mathrm{p}<0.01)$, FDS $(\mathrm{p}<0.01)$, PAS $(\mathrm{p}<0.01)$, and lower ASHA NOMS $(\mathrm{p}=0.02)$ at initial evaluation. However, there was no significant difference for the swallowing functions between benign and malignant brain tumor patients.

Conclusion Swallowing function of brain tumor patients was not different from that of stroke patients according to matching age, location of lesion, and functional status. Similar to the stroke patients, brain tumor patients with infratentorial lesions present poor swallowing functions. However, the type of brain tumor as malignancy does not influence swallowing functions.

Keywords Brain tumor, Dysphagia, Videofluoroscopic swallowing study, Infratentorial lesion, Malignancy

Department of Rehabilitation Medicine, Asan Medical Center, University of Ulsan College of Medicine, 88 Olympic-ro 43-gil, Songpa-gu, Seoul 138736 , Korea

Tel: +82-2-3010-3800, Fax: +82-2-3010-6964, E-mail: mhchun@amc.seoul.kr

(c) This is an open-access article distributed under the terms of the Creative Commons Attribution Non-Commercial License (http://creativecommons.org/ licenses/by-nc/3.0) which permits unrestricted noncommercial use, distribution, and reproduction in any medium, provided the original work is properly cited. Copyright $\odot 2013$ by Korean Academy of Rehabilitation Medicine 


\section{INTRODUCTION}

Symptoms of brain tumors are highly variable with weaknesses, cognitive impairments, fatigues, mood disorders, seizures, and headaches all being observed [1]. These symptoms depend on the tumor locations and sizes [2]. Dysphagia, with a frequency of about $63 \%$, is also a common symptom found in brain tumor patients [1]. And dysphagia in brain tumor patients is a risk factor for complications, such as aspiration pneumonia, dehydration, and malnutrition, which impacts on quality of life and on the long-term prognosis in these cases [3].

There were only few studies on dysphagia for brain tumor patients. In the previous study conducted by Wesling et al. [2], functional dysphagia outcomes from brain tumor patients were similar to that of stroke patients who matched according to age, functional status, and site of lesion. But, there was only one brain tumor patient with brainstem lesion in this study, thus, the swallowing function of brain tumor patients with infratentorial lesion was not thoroughly evaluated. In stroke patients, different patterns of dysphagia are also found according to brain lesion location. Discoordination of the tongue, oropharynx, and laryngopharynx predominate in the cortical and subcortical lesions, whereas the incomplete laryngeal closure and failure of cricopharyngeal muscle relaxation predominate in brainstem lesions [4]. It was assumed that the swallowing function of brain tumor patients with infratentorial lesions are different from those with supratentorial lesions swallowing, however, no study has yet addressed the differences of swallowing function patterns according to lesion locations in brain tumor patients. Additionally, whether the brain tumor was benign or malignant as the determinant of swallowing function was not investigated. So, further study is needed to include more numbers of infratentorial brain tumor patients and to evaluate the major determinant of swallowing function in brain tumor patients.

Thus, the first aim of this study was to compare dysphagia patterns and clinical outcomes between brain tumor and stroke patients. Second, we investigate the different swallowing functions according to brain lesion location and the type of benign or malignant tumor in brain tumor patients.

\section{MATERIALS AND METHODS}

\section{Subjects}

This was designed as a retrospective study and we collected information from medical chart review. Our facility's Institutional Review Board approved the study protocol. All brain tumor patients were recruited from an inpatient rehabilitation unit from January 2009 to March 2012. The following malignant or benign brain tumor patients were included: patients whose swallowing function was evaluated by a videofluoroscopic swallowing study (VFSS) and patients who had definite evidence of brain tumor on magnetic resonance imaging (MRI) or computerized tomography (CT). Interpretations of brain MRI or CT data were performed by a radiology specialist.

The locations of the brain lesions in our current study were classified as either supratentorial or infratentorial. If the brain lesion could not be classified in this way due to the large size or number, the patient was excluded from our analysis. The same number of acute stroke patients (less than 6 weeks post-onset) who matched by age ( \pm 5 years), sex (male or female), location of lesion (supratentorial or infratentorial), and initial Korean version of the Modified Barthel Index (K-MBI, \pm 5 points) score, were recruited for comparison. It was reported that differences in swallowing functions were observed according to whether the lesion was left or right [5]. Therefore, brain tumor patients were also matched with stroke patients according to the side of lesion.

\section{Videofluoroscopic swallowing study}

VFSS was performed during the initial 3 days of admission to the rehabilitation unit. Swallowing images in the lateral projection were acquired while the subjects were seated. A modified version of the protocol from the study of Logemann [6] was used. First, $3 \mathrm{~mL}$ of thick liquid was provided to the subjects. Next, swallowing tests involving $3 \mathrm{~mL}$ of pureed, semi-solid, solid, and thin liquids were performed in this sequence. All food samples contained barium and each test was performed three times. If there was a large amount of aspiration, the study was aborted and the patients were encouraged to expectorate the food materials. All test procedures were recorded and the findings were analyzed by two physiatrists with expertise in rehabilitation medicine. If there was any abnormal finding during oral, pharyngeal, or esophageal phases, the 
patient was diagnosed with dysphagia.

\section{Evaluation}

Swallowing function was evaluated during the initial 3 days of admission. The American Speech-LanguageHearing Association (ASHA) National Outcome Measurement System (NOMS) swallowing scale and the clinical dysphagia scale (CDS) were used to evaluate the study subjects. VFSS was used to evaluate the functional dysphagia scale (FDS), penetration-aspiration scale (PAS) [7], oral transit time (OTT), pharyngeal transit time (PTT), and the presence of vallecular pouch residue, pyriform sinus residue, laryngopharyngeal incoordination, premature spillage, and any decrease in the swallowing reflex.

The ASHA NOMS swallowing scale is a multi-dimensional tool designed to measure both the diet level and the supervision level required by assigning a single number between 1 and 7 [8]. The CDS consists of eight rating items (lesion location, tracheostomy, history of aspiration, lip sealing, chewing and mastication, tongue protrusion, laryngeal elevation, and reflex coughing) [9]. The FDS is a scale which was developed to quantify dysphagia severity [10], and PAS evaluates airway invasions [11]. A higher ASHA NOMS score indicates a better swallowing function, whereas higher CDS, FDS, and PAS scores indicate a poorer swallowing function. The OTT was measured as the first backward movement of the bolus from when the head of the bolus passed the point of the ramus of the mandible until the tail of the bolus left the cricopharyngeal region. The PTT was measured from the time the head of the bolus passed the ramus of the mandible until the onset of laryngeal elevation [12].

Initial diets and supervision levels were documented before VFSS, and follow-up ASHA NOMS at discharge were documented within the 24-hour period prior to discharge. Following VFSS, patients who have dysphagia underwent swallowing therapy for 30 minutes a day, 5 days a week, more than two weeks. Types of dysphagia treatments were compensatory treatments (positioning strategies, diet modification, or feeding strategies), strengthening exercises and combination of both. Data on pneumonia occurrence and the feeding method at discharge were obtained through a medical chart review. For comparison on the functional status of the subjects, we evaluated the K-MBI at about the same time as the VFSS. The results were compared according to brain le- sion location and type of brain injury.

\section{Statistical analysis}

Mann-Whitney U test was used to compare the age, disease duration, OTT, PTT, ASHA NOMS, CDS, FDS, and PAS results between the brain tumor and stroke groups. A chi-square test was used for comparing the vallecular pouch residue, pyriform sinus residue, laryngopharyngeal incoordination, premature spillage, decreased swallowing reflex, occurrence of pneumonia, and the feeding method at discharge. The same test was used to compare parameters according to lesion location and whether the lesion was benign or malignant. Results were considered statistically significant if the p-value was less than 0.05 . Statistical analysis was performed with SPSS ver. 18.0 (SPSS Inc., Chicago, IL, USA) for Windows statistical software.

\section{RESULTS}

From January 2009 to June 2012, a total of 127 brain tumor patients were admitted to the rehabilitation medicine department. Of those patients, VFSS was performed in 58 cases. Eighteen patients were excluded: 14 had too large or too many lesions, which complicated the lesion classifications as either supratentorial or infratentorial, and four patients had ischemic or hemorrhagic strokes. Thus, a total of 40 brain tumor patients participated in our study and 40 age-, sex-, and K-MBI-matching stroke patients were also enrolled as the stroke group for comparisons with the brain tumor group.

\section{Demographic data}

Demographic characteristics were demonstrated in Table 1. In both groups, approximately half of the subjects were males $(55.0 \%)$ and had supratentorial lesions $(47.5 \%)$. The mean age of the brain tumor group was $52.1 \pm 15.7$ years (range, 19 to 74 years) whereas that of the stroke group was $51.2 \pm 11.8$ years (range, 24 to 74 years). The mean K-MBI scores for the brain tumor and stroke groups were $41.9 \pm 31.7$ and $42.9 \pm 25.6$, respectively. The lengths of stay in rehabilitation units for the brain tumor and stroke groups were $27.4 \pm 3.7$ and $26.2 \pm 4.9$ days. The mean disease durations for the brain tumor and stroke groups were $7.7 \pm 13.4$ and $0.7 \pm 1.7$ months, respectively $(p<0.05)$. Disease duration of brain tumor patients were 
longer than stroke patients, because brain tumor patients required more time for diagnosis and treatments, such as surgery, chemotherapy, and radiation therapies before starting the rehabilitation. Age and K-MBI scores were not found to be significantly different.

Table 1. Demographic characteristics of the brain tumor group and stroke group

\begin{tabular}{lcc}
\hline \multicolumn{1}{c}{ Characteristic } & $\begin{array}{c}\text { Brain tumor } \\
(\mathbf{n = 4 0 )}\end{array}$ & Stroke $(\mathbf{n = 4 0 )}$ \\
\hline Age (yr) & $52.1 \pm 15.7$ & $51.2 \pm 11.8$ \\
\hline $\begin{array}{l}\text { Gender (male:female) } \\
\text { Location of lesion }\end{array}$ & $22: 18$ & $22: 18$ \\
$\quad$ Supratentorial & $19(47.5)$ & $19(47.5)$ \\
\hline$\quad$ Infratentorial & $21(52.5)$ & $21(52.5)$ \\
\hline K-MBI & $41.9 \pm 31.7$ & $42.9 \pm 25.6$ \\
\hline $\begin{array}{l}\text { Duration of disease } \\
\text { (mo) }\end{array}$ & $7.7 \pm 13.4^{*}$ & $0.7 \pm 1.7^{*}$ \\
\hline $\begin{array}{l}\text { Lengths of stay in reha- } \\
\text { bilitation unit (day) }\end{array}$ & $27.4 \pm 3.7$ & $26.2 \pm 4.9$ \\
\hline
\end{tabular}

Values are presented as mean \pm standard deviation or number (\%).

K-MBI, initial Korean version of Modified Barthel Index. ${ }^{*} \mathrm{p}<0.05$.
In accordance with the 2007 World Health Organization classification for tumors of the central nervous system [13], 16 of our brain tumor patient subjects had a malignant brain tumor, such as glioma (7 patients), metastatic brain tumor (5), astrocytoma (5), mixed germ cell tumor (1), and ependymoma (1), whereas 24 of our brain tumor patients had benign brain lesions, such as meningioma (16), schwannoma (6), and craniopharyngioma (2). From the 35 out of 40 brain tumor patients in our present cohort underwent tumor resection, 13 received chemotherapy, and 14 were treated with radiation therapy prior to the commencement of the present study. There were no significant demographic differences between the two study groups.

Differences in dysphagia between brain tumor and stroke patients

Dysphagia was noted using VFSS in $72.5 \%$ of our brain tumor patients and $77.5 \%$ of our stroke patients. There were no significant differences found in the numbers suffering from dysphagia, or between the initial ASHA NOMS, FDS, CDS, PAS, OTT, PTT, and other VFSS findings (Table 2). Furthermore, at the time of discharge, same number of patients was following a regular diet in

Table 2. Comparisons of swallowing functions between brain tumor group and stroke group

\begin{tabular}{lccc}
\hline & Brain tumor $(\mathbf{n}=\mathbf{4 0})$ & Stroke $(\mathbf{n = 4 0 )}$ & p-value \\
\hline Proportion of dysphagia & $29(72.5)$ & $31(77.5)$ & 0.80 \\
Initial ASHA NOMS & $3.9 \pm 2.4$ & $4.6 \pm 1.9$ & 0.25 \\
Functional dysphagia scale & $8.3 \pm 10.6$ & $5.8 \pm 7.1$ & 0.33 \\
Clinical dysphagia scale & $27.1 \pm 12.8$ & $28.8 \pm 15.8$ & 0.95 \\
Penetration aspiration scale & $3.7 \pm 2.9$ & $4.8 \pm 2.7$ & 0.12 \\
Oral transit time (sec) & $0.4 \pm 0.3$ & $0.5 \pm 0.8$ & 0.15 \\
Pharyngeal transit time (sec) & $0.4 \pm 0.2$ & $0.5 \pm 0.4$ & 0.87 \\
Vallecular pouch residue & $15(37.5)$ & $14(35.0)$ & 1.00 \\
Pyriform sinus residue & $12(30.0)$ & $10(25.0)$ & 0.80 \\
Decreased swallowing reflex & $22(55.0)$ & $21(52.5)$ & 1.00 \\
Premature spillage & $4(10.0)$ & $5(12.5)$ & 1.00 \\
Laryngopharyngeal incoordination & $8(20.0)$ & $7(17.5)$ & 1.00 \\
Occurrence of pneumonia & $5(12.5)$ & $5(12.5)$ & 1.00 \\
Regular diets at discharge & $25(62.5)$ & $25(62.5)$ & 1.00 \\
Follow-up of ASHA NOMS & $5.4 \pm 2.4$ & $5.4 \pm 2.5$ & 0.92 \\
\hline
\end{tabular}

Values are presented as mean \pm standard deviation or number (\%).

There was no significant difference in all parameters between two groups.

ASHA NOMS, the American Speech-Language-Hearing Association National Outcome Measurement System swallowing scale. 
Table 3. Comparisons of swallowing function in patients with infratentorial lesion

\begin{tabular}{lccc}
\hline & Brain tumor $(\mathbf{n = 2 1})$ & Stroke $(\mathbf{n = 2 1})$ & p-value \\
\hline Proportion of dysphagia & $19(90.5)$ & $18(85.7)$ & 1.00 \\
Initial ASHA NOMS & $3.1 \pm 2.1$ & $3.4 \pm 2.0$ & 0.67 \\
Functional dysphagia scale & $14.2 \pm 12.0$ & $13.1 \pm 16.6$ & 0.88 \\
Clinical dysphagia scale & $25.6 \pm 14.1$ & $32.7 \pm 16.0$ & 0.31 \\
Penetration aspiration scale & $5.2 \pm 2.6$ & $5.4 \pm 2.6$ & 0.88 \\
Oral transit time (sec) & $0.4 \pm 0.1$ & $0.7 \pm 0.8$ & 0.12 \\
Pharyngeal transit time (sec) & $0.5 \pm 0.3$ & $0.6 \pm 0.4$ & 0.81 \\
Vallecular pouch residue & $14(66.7)$ & $13(61.9)$ & 1.00 \\
Pyriform sinus residue & $12(57.1)$ & $10(47.6)$ & 0.76 \\
Decreased swallowing reflex & $17(80.9)$ & $16(76.2)$ & 1.00 \\
Premature spillage & $2(9.5)$ & $3(9.5)$ & 1.00 \\
Laryngopharyngeal incoordination & $6(28.6)$ & $5(23.8)$ & 1.00 \\
\hline Occurrence of pneumonia & $2(9.5)$ & $3(14.3)$ & 1.00 \\
Regular diets at discharge & $9(42.9)$ & $10(47.6)$ & 1.00 \\
Follow-up of ASHA NOMS & $5.0 \pm 2.2$ & $4.6 \pm 2.4$ & 0.89 \\
\hline
\end{tabular}

Values are presented as mean \pm standard deviation or number (\%).

There was no significant difference in all parameters between two groups.

ASHA-NOMS, the American Speech-Language-Hearing Association National Outcome Measurement System swallowing scale.

Table 4. Comparisons of swallowing functions and characteristics according to location of brain tumor

\begin{tabular}{lccc}
\hline & Supratentorial lesion $(\mathbf{n = 1 9})$ & Infratentorial lesion $(\mathbf{n}=\mathbf{2 1})$ & p-value \\
\hline Age (yr) & $55.9 \pm 15.1$ & $48.6 \pm 15.4$ & 0.15 \\
Proportion of dysphagia & $10(52.6)$ & $19(90.5)$ & $0.01^{*}$ \\
Initial ASHA NOMS & $4.7 \pm 2.5$ & $3.1 \pm 2.1$ & $0.02^{*}$ \\
Functional dysphagia scale & $1.8 \pm 1.6$ & $14.2 \pm 12.0$ & $<0.01^{*}$ \\
Clinical dysphagia scale & $28.8 \pm 12.3$ & $25.6 \pm 14.1$ & 0.25 \\
Penetration aspiration scale & $1.9 \pm 2.2$ & $5.2 \pm 2.6$ & $<0.01^{*}$ \\
Oral transit time (sec) & $0.4 \pm 0.3$ & $0.4 \pm 0.1$ & 0.94 \\
Pharyngeal transit time (sec) & $0.3 \pm 0.2$ & $0.5 \pm 0.3$ & 0.25 \\
Vallecular pouch residue & $1(5.3)$ & $14(66.7)$ & $<0.01^{*}$ \\
Pyriform sinus residue & $0(0.0)$ & $12(57.1)$ & $<0.01^{*}$ \\
Decreased swallowing reflex & $5(26.3)$ & $17(80.9)$ & $<0.01^{*}$ \\
Premature spillage & $2(10.5)$ & $2(9.5)$ & 0.66 \\
Laryngopharyngeal incoordination & $2(10.5)$ & $6(28.6)$ & 0.15 \\
Occurrence of pneumonia & $3(15.8)$ & $2(9.5)$ & 0.45 \\
Regular diets at discharge & $16(84.2)$ & $9(42.9)$ & 0.14 \\
\hline Follow-up of ASHA NOMS & $5.7 \pm 2.3$ & $5.0 \pm 2.2$ & 0.08 \\
\hline K-MBI & $35.9 \pm 31.1$ & $47.3 \pm 31.6$ & 0.27 \\
\hline
\end{tabular}

Values are presented as mean \pm standard deviation or number (\%).

ASHA-NOMS, the American Speech-Language-Hearing Association National Outcome Measurement System swallowing scale; K-MBI, initial Korean version of Modified Barthel Index.

${ }^{*} \mathrm{p}<0.05$. 
both groups. Follow-up of ASHA NOMS results and the frequency of pneumonia were also observed to be similar. In addition, when we compared infratentorial brain tumor patients with infratentorial stroke patients, there were no significant differences in all parameters between these patients (Table 3).

\section{Dysphagia according to location of brain tumor}

There was a significant difference found in the frequency of dysphagia according to lesion locations (Table 4). In the 19 brain tumor patients with supratentorial lesions, 10 patients (52.6\%) showed dysphagia, whereas in the 21 patients with infratentorial lesions, 19 (90.5\%) showed dysphagia $(\mathrm{p}=0.01)$. Compared with brain tumor patients who have supratentorial lesions, brain tumor patients who have infratentorial lesions showed higher proportions of dysphagia $(\mathrm{p}=0.01)$, vallecular pouch residue $(\mathrm{p}=0.01)$, pyriform sinus residue $(\mathrm{p}=0.01)$, FDS $(\mathrm{p}<001)$, PAS $(\mathrm{p}<0.01)$, and lower ASHA NOMS $(\mathrm{p}=0.02)$. Although the outcome measured at discharge regarding the portion of regular diets seemed to be poorer in patients with infratentorial lesions, it was not statistically significant $(\mathrm{p}=0.14)$. At the time of discharge, although $84.2 \%$ of supratentorial lesion patients were following a regular diet, only $42.9 \%$ of infratentorial lesion patients were able to eat a regular diet. On the other hand, there were no significant differences in the CDS, OTT, PTT, in the proportion of premature spillage, in laryngopharyngeal incoordination, K-MBI, and ASHA NOMS at discharge between the two groups.

\section{Dysphagia according to whether the tumor was benign or malignant}

In our present study, there were no significant differences found between the benign and malignant groups in the initial ASHA NOMS (4.1 \pm 2.5 vs. $3.5 \pm 2.2$, respectively; $\mathrm{p}=0.33$ ), FDS ( $9.5 \pm 10.0$ vs. $6.4 \pm 11.5$, respectively; $\mathrm{p}=0.24$ ), CDS (26.5 \pm 13.7 vs. $27.9 \pm 11.7$, respectively; $\mathrm{p}=0.71$ ), or PAS (3.2 \pm 2.7 vs. $4.4 \pm 3.0$, respectively; $\mathrm{p}=0.20$ ) scores (Table 5). The OTT and PTT scores, degrees of post swallowing residue, levels of diet modification at discharge, and prevalence of pneumonia were also not significantly

Table 5. Comparisons of swallowing functions and characteristics according to the type of a brain tumor as benign or malignant

\begin{tabular}{lccc}
\hline & Benign (n=24) & Malignant (n=16) & p-value \\
\hline Age (yr) & $54.0 \pm 14.7$ & $49.2 \pm 17.2$ & 0.49 \\
Proportion of dysphagia & $17(70.8)$ & $12(75.0)$ & 1.00 \\
Initial ASHA NOMS & $4.1 \pm 2.5$ & $3.5 \pm 2.2$ & 0.33 \\
Functional dysphagia scale & $9.5 \pm 10.0$ & $6.4 \pm 11.5$ & 0.24 \\
\hline Clinical dysphagia scale & $26.5 \pm 13.7$ & $27.9 \pm 11.7$ & 0.71 \\
\hline Penetration aspiration scale & $3.2 \pm 2.7$ & $4.4 \pm 3.0$ & 0.20 \\
\hline Oral transit time (sec) & $0.4 \pm 0.3$ & $0.4 \pm 0.2$ & 0.32 \\
Pharyngeal transit time (sec) & $0.4 \pm 0.2$ & $0.5 \pm 0.3$ & 0.37 \\
Vallecular pouch residue & $9(37.5)$ & $6(37.5)$ & 1.00 \\
Pyriform sinus residue & $8(33.3)$ & $4(25.0)$ & 0.73 \\
Decreased swallowing reflex & $13(54.2)$ & $9(56.3)$ & 1.00 \\
Premature spillage & $1(4.2)$ & $3(18.8)$ & 0.28 \\
\hline Laryngopharyngeal incoordination & $5(20.8)$ & $3(18.8)$ & 1.00 \\
Occurrence of pneumonia & $2(8.3)$ & $3(18.8)$ & 0.37 \\
Regular diets at discharge & $18(75.0)$ & $7(43.8)$ & 0.09 \\
\hline Follow-up of ASHA NOMS & $5.7 \pm 2.3$ & $4.8 \pm 2.5$ & 0.17 \\
\hline K-MBI & $45.0 \pm 32.6$ & $37.3 \pm 30.7$ & 0.37 \\
\hline Val
\end{tabular}

Values are presented as mean \pm standard deviation or number (\%).

There was no significant difference in all parameters between two groups.

ASHA NOMS, the American Speech-Language-Hearing Association National Outcome Measurement System swallowing scale; K-MBI, initial Korean version of Modified Barthel Index. 
different in the benign and malignant groups.

\section{DISCUSSION}

This study evaluates that the swallowing functions of brain tumor patients when compared with stroke patients of matching lesion locations. In previous study, comparisons of functional status and outcomes between brain tumor patients and stroke patients were performed [14]. In their study, the total functional independence measure was similar in two groups at admission, whereas the mobility was good in brain tumor group. However, there were insufficient studies on swallowing functions of brain tumor patients.

Dysphagia was found in many brain tumor patients in this study. Wesling et al. [2] reported that there were no differences in the ASHA NOMS at admission between brain tumor patients and stroke patients who matched according to age, location of lesion, and functional status. And, functional dysphagia gains can be achieved for brain tumor patients undergoing inpatient rehabilitations through the same types and intensities of swallowing rehabilitation following a stroke in this study. Although the brain tumor and stroke patients in their study matched by onset age, site of lesion, and functional status, a comparison of dysphagia according to the lesion location or whether the lesion was benign or malignant was not performed. We tried to classify brain tumor patients by locations of lesion and the types of benign or malignant tumor in our current study, and then, compared patients according to these factors. And only one patient have infratentorial lesion in their study, thus, we conducted a study including more numbers of patients with infratentorial lesions.

In the previous study by Lee and Kim [4], brain lesion locations in stroke patients were classified as cortical, subcortical, or brainstem. Stroke lesions, caused by the ruptures or occlusions of cerebral vessels, are either classified as cortical, subcortical, or brainstem according to the supplying vessels (e.g., internal carotid artery, middle cerebral artery). However, it is difficult to use the same categories for brain tumors as they are not related to vascular territory, and this impedes the clear regional separations of these lesions. It is also difficult to classify a lesion as either cortical or subcortical. In previous studies, brain tumors were classified as either supratento- rial or infratentorial lesions $[7,15,16]$. In those reports, the symptoms and types of common brain tumors were found to differ according to locations. Commonly, a brain lesion location is classed as supratentorial or infratentorial.

In our present study, patients with infratentorial brain tumor lesions showed poor swallowing functions, which are similar to stroke patients with brainstem lesions. On the other hand, there were no differences in follow-ups of ASHA NOMS at discharge between supratentorial brain tumor patients and infratentorial brain tumor patients. As treated by swallowing therapy and chemoradiothrapy, swallowing function was improved in most supratentorial and infratentorial patients. However, three patients with supratentorial lesions and four patients with infratentorial lesions were not improved at all. Proportion of patients who were refractory to therapy was similar in two groups, so follow-up of ASHA NOMS was similar.

In the present study, there were no differences found in the swallowing functions between benign and malignant brain tumor patients. Furthermore, there were also no differences for functional status in the K-MBI results, the ASHA NOMS, FDS, CDS findings, and the frequency of pneumonia were not significantly different between benign and malignant brain tumor patients. These results suggest that a brain tumor malignancy is not an important predictive factor for dysphagia, whereas the brain tumor location is a major determinant of swallowing function. And there were no significant differences on swallowing functions between brain tumor and stroke patients who matched by age, lesion location, and functional status. These findings suggest that whether the type of brain injury was brain tumor or stroke is not an important factor when determining dysphagia characteristics.

Our study has some limitations. First, we matched patients by lesion locations, but the sizes and durations of the lesions were not matched. When nervous system was injured, regardless of etiology, the early rehabilitation improved functional outcomes [17]. In our study, brain tumor patients have longer duration periods than stroke patients, so, it is possible that the difference of disease durations influence the clinical outcomes. Second, treatment method of brain tumor patients may influence swallowing functions. In the present study, 35 brain tumor patients had surgeries. Almost all of them showed 
postoperative changes, such as fluid collection, hemorrhage, edema, and pneumocephalus. Out of 14 patients who were treated with radiotherapy, 3 patients were diagnosed with radiation-induced brain injury, and they received steroid therapies to control brain edema. These operation or radiation induced changes can influence symptoms of brain tumor patients [18-21]. And thirteen brain tumor patients received chemotherapy with agents, such as cisplatin, carboplatin, temozolomide, paclitaxel, and etoposide. It has been known that most of these agents can induce neuropathy [22,23]. Our study was also performed at a single institution and a small number of patients participated. Future studies that involve a larger cohort and controls for other disease factors are necessary.

In conclusion, swallowing function of brain tumor patients was not different from that of stroke patients when matched by age, location of lesion, and functional status. And the different patterns of dysphagia found in brain tumor patients were related to the location of the lesions. On the other hand, it seems that whether the tumor is benign or malignant is not a major determinant of the swallowing function for brain tumor patients. Further studies are needed for investigating the effects of lesion size and treatment methods for swallowing functions of brain tumor patients in large population.

\section{CONFLICT OF INTEREST}

No potential conflict of interest relevant to this article was reported.

\section{REFERENCES}

1. Vargo M. Brain tumor rehabilitation. Am J Phys Med Rehabil 2011;90(5 Suppl 1):S50-62

2. Wesling M, Brady S, Jensen M, Nickell M, Statkus D, Escobar N. Dysphagia outcomes in patients with brain tumors undergoing inpatient rehabilitation. Dysphagia 2003;18:203-10.

3. Perlman AL. Dysphagia in stroke patients. Semin Neurol 1996;16:341-8.

4. Lee CK, Kim JA. Pattern of post-stroke swallowing disorder according to the brain lesion. J Korean Acad Rehabil Med 2001;25:193-201.

5. Robbins J, Levine RL, Maser A, Rosenbek JC, Kemp- ster GB. Swallowing after unilateral stroke of the cerebral cortex. Arch Phys Med Rehabil 1993;74:1295-300.

6. Logemann JA. Manual for the videofluorographic study of swallowing. 2nd ed. Austin: Pro-Ed; 1993.

7. Pietila S, Korpela R, Lenko HL, Haapasalo H, Alalantela R, Nieminen P, et al. Neurological outcome of childhood brain tumor survivors. J Neurooncol 2012;108:153-61.

8. O'Neil KH, Purdy M, Falk J, Gallo L. The dysphagia outcome and severity scale. Dysphagia 1999;14:13945.

9. Han TR, Paik NJ, Park JW. The clinical functional scale for dysphagia in stroke patients. Korean J Stroke 2001;3:153-7.

10. Han TR, Paik NJ, Park JW. Quantifying swallowing function after stroke: a functional dysphagia scale based on videofluoroscopic studies. Arch Phys Med Rehabil 2001;82:677-82.

11. Rosenbek JC, Robbins JA, Roecker EB, Coyle JL, Wood JL. A penetration-aspiration scale. Dysphagia 1996;11:93-8.

12. Lin CW, Chang YC, Chen WS, Chang K, Chang HY, Wang TG. Prolonged swallowing time in dysphagic Parkinsonism patients with aspiration pneumonia. Arch Phys Med Rehabil 2012;93:2080-4.

13. Louis DN, Ohgaki H, Wiestler OD, Cavenee WK, Burger PC, Jouvet A, et al. The 2007 WHO classification of tumours of the central nervous system. Acta Neuropathol 2007;114:97-109.

14. Huang ME, Cifu DX, Keyser-Marcus L. Functional outcome after brain tumor and acute stroke: a comparative analysis. Arch Phys Med Rehabil 1998;79:1386-90.

15. Battistella PA, Ruffilli R, Viero F, Bendagli B, Condini A. Brain tumors: classification and clinical aspects. Pediatr Med Chir 1990;12:33-9.

16. Purdy RA, Kirby S. Headaches and brain tumors. Neurol Clin 2004;22:39-53.

17. Brown JM, Deriso DM, Tansey KE. From contemporary rehabilitation to restorative neurology. Clin Neurol Neurosurg 2012;114:471-4.

18. Wang XF, Zhang S, Ye YH, Chen YP, Liu XY. Clinicopathologic features of delayed radiation-induced brain injury after radiotherapy for brain tumor. Zhonghua Bing Li Xue Za Zhi 2012;41:224-8.

19. Ouyang M, McDonagh DL, Phillips-Bute B, James ML, Friedman AH, Gan TJ. Does midline shift predict post- 
operative nausea in brain tumor patients undergoing awake craniotomy? A retrospective analysis. Curr Med Res Opin 2013;29:1033-8.

20. Bebawy JF. Perioperative steroids for peritumoral intracranial edema: a review of mechanisms, efficacy, and side effects. J Neurosurg Anesthesiol 2012;24:1737.

21. Kim YZ, Kim DY, Yoo H, Yang HS, Shin SH, Hong EK, et al. Radiation-induced necrosis deteriorating neuro- logical symptoms and mimicking progression of brain metastasis after stereotactic-guided radiotherapy. Cancer Res Treat 2007;39:16-21.

22. Sghirlanzoni A, Silvani A, Scaioli V, Pareyson D, Marchesan R, Boiardi A. Cisplatin neuropathy in brain tumor chemotherapy. Ital J Neurol Sci 1992;13:311-5.

23. Amato AA, Collins MP. Neuropathies associated with malignancy. Semin Neurol 1998;18:125-44. 